PROCEEDINGS OF THE

AMERICAN MATHEMATICAL SOCIETY

Volume 133, Number 4, Pages 1047-1056

S 0002-9939(04)07819-0

Article electronically published on November 19, 2004

\title{
SUBELLIPTIC CORDES ESTIMATES
}

\author{
ANDRÁS DOMOKOS AND JUAN J. MANFREDI
}

(Communicated by David S. Tartakoff)

\begin{abstract}
We prove Cordes type estimates for subelliptic linear partial differential operators in non-divergence form with measurable coefficients in the Heisenberg group. As an application we establish interior horizontal $W^{2,2}$ regularity for p-harmonic functions in the Heisenberg group $\mathbb{H}^{1}$ for the range $\frac{\sqrt{17}-1}{2} \leq p<\frac{5+\sqrt{5}}{2}$.
\end{abstract}

\section{INTRODUCTION}

The main goal of this paper is to prove some estimates of Cordes type for subelliptic partial differential operators in non-divergence form with measurable coefficients in the Heisenberg group, including the linearized p-Laplacian. To show the applicability of our methods let us state the following theorem that constitutes a special case of our results.

Theorem 1.1. Let $\frac{\sqrt{17}-1}{2} \leq p<\frac{5+\sqrt{5}}{2}$. Then any $p$-harmonic function in the Heisenberg group $\mathbb{H}^{1}$ initially in $H W_{\mathrm{loc}}^{1, p}$ is in $H W_{\text {loc }}^{2,2}$.

We build on previous regularity results obtained by Marchi [7, 8] and extended by the first author [3, which give non-uniform bounds of the $H W^{2,2}$ (or $H W^{2, p}$ ) norm of the approximate p-harmonic functions. Using the Cordes condition 2, 11] and Strichartz's spectral analysis [10] we establish $H W^{2,2}$ estimates for linear subelliptic partial differential operators with measurable coefficients. As an application we obtain uniform $H W^{2,2}$ bounds for the approximate p-harmonic functions for $\mathrm{p}$ in a range that depends on the dimension of the Heisenberg group $\mathbb{H}^{n}$.

Consider the Heisenberg group $\mathbb{H}^{n}$, that is, $\mathbb{R}^{2 n+1}$ with the group multiplication $\left(x_{1}, \ldots, x_{2 n}, t\right) \cdot\left(y_{1}, \ldots, y_{2 n}, u\right)=\left(x_{1}+y_{1}, \ldots, x_{2 n}+y_{2 n}, t+u-\frac{1}{2} \sum_{i=1}^{n}\left(x_{i} y_{n+i}-x_{n+i} y_{i}\right)\right)$.

For $i \in\{1, \ldots, n\}$ consider the vector fields

$$
X_{i}=\frac{\partial}{\partial x_{i}}-\frac{x_{n+i}}{2} \frac{\partial}{\partial t}, \quad X_{n+i}=\frac{\partial}{\partial x_{n+i}}+\frac{x_{i}}{2} \frac{\partial}{\partial t}, T=\frac{\partial}{\partial t} .
$$

The nontrivial commutators are $\left[X_{i}, X_{n+i}\right]=T$; otherwise $\left[X_{i}, X_{j}\right]=0$.

Received by the editors August 13, 2003.

2000 Mathematics Subject Classification. Primary 35H20, 35J70.

Key words and phrases. Cordes conditions, subelliptic equations, p-Laplacian.

The authors were partially supported by NSF award DMS-0100107.

(C)2004 American Mathematical Society Reverts to public domain 28 years from publication 
Let $\Omega \subset \mathbb{H}^{n}$ be a domain. Consider the following Sobolev space with respect to the horizontal vector fields $X_{i}$ as

$$
H W^{2,2}(\Omega)=\left\{u \in L^{2}(\Omega): X_{i} X_{j} u \in L^{2}(\Omega) \text {, for all } i, j \in\{1, \ldots, 2 n\}\right\}
$$

endowed with the inner product

$$
(u, v)_{H W^{2,2}(\Omega)}=\int_{\Omega}\left(u(x) v(x)+\sum_{i, j=1}^{2 n} X_{i} X_{j} u(x) \cdot X_{i} X_{j} v(x)\right) d x .
$$

$H W^{2,2}(\Omega)$ is a Hilbert space and let $H W_{0}^{2,2}(\Omega)$ be the closure of $C_{0}^{\infty}(\Omega)$ in this Hilbert space.

We denote by $X^{2} u$ the matrix of second-order horizontal derivatives whose entries are $\left(X^{2} u\right)_{i j}=X_{j}\left(X_{i} u\right)$, and by $\Delta_{H} u=\sum_{i=1}^{2 n} X_{i} X_{i} u$ the subelliptic Laplacian associated to the horizontal vector fields $X_{i}$.

Lemma 1.1. For all $u \in H W_{0}^{2,2}(\Omega)$ we have

$$
\left\|X^{2} u\right\|_{L^{2}(\Omega)} \leq c_{n}\left\|\Delta_{H} u\right\|_{L^{2}(\Omega)},
$$

where

$$
c_{n}=\sqrt{1+\frac{2}{n}} .
$$

The constant $c_{n}$ is sharp when $\Omega=\mathbb{H}^{n}$.

Proof. We follow the spectral analysis of $\Delta_{H}$ developed by Strichartz [10]. Let us recall the fact that $-\Delta_{H}$ and $i T$ commute and share the same system of eigenvectors

$$
\begin{aligned}
\Phi_{\lambda, k, l}(z, t)= & \frac{\lambda^{n}}{(2 \pi)^{n+1}(n+2 k)^{n+1}} \cdot \exp \left(-\frac{i l \lambda t}{n+2 k}\right) \\
& \cdot \exp \left(-\frac{\lambda|z|^{2}}{4(n+2 k)}\right) \cdot L_{k}^{n-1}\left(\frac{\lambda|z|^{2}}{2(n+2 k)}\right),
\end{aligned}
$$

where $l= \pm 1, k \in\{0,1,2, \ldots\}$ and $L_{k}^{n-1}$ is the Laguerre polynomial

$$
L_{k}^{n-1}(t)=\frac{e^{t}}{t^{n-1}} \cdot \frac{1}{k !} \cdot \frac{d^{k}}{d t^{k}}\left(e^{-t} t^{k+n-1}\right) .
$$

For the eigenvalues, we have the following relations:

$$
\begin{aligned}
i T u * \Phi_{\lambda, k, l} & =\frac{l \lambda}{n+2 k} u * \Phi_{\lambda, k, l}, \\
-\Delta_{H} u * \Phi_{\lambda, k, l} & =\lambda u * \Phi_{\lambda, k, l},
\end{aligned}
$$

where $*$ denotes the group convolution. Therefore, the spectral decomposition of $\Delta_{H} u$ for $u \in C_{0}^{\infty}(\Omega)$, the Plancherel formula, and relations (1.1)-(1.2) give

$$
\begin{aligned}
\left\|\Delta_{H} u\right\|_{L^{2}(\Omega)}^{2} & =2 \pi \sum_{k=0}^{\infty} \sum_{l= \pm 1}(n+2 k) \int_{0}^{\infty} \int_{\mathbb{C}^{n}}\left|\Delta_{H} u * \Phi_{\lambda, k, l}(z, 0)\right|^{2} d z d \lambda \\
& =2 \pi \sum_{k=0}^{\infty} \sum_{l= \pm 1}(n+2 k) \int_{0}^{\infty} \int_{\mathbb{C}^{n}}\left|\frac{n+2 k}{l} i T u * \Phi_{\lambda, k, l}(z, 0)\right|^{2} d z d \lambda \\
& \geq n^{2}\|T u\|_{L^{2}}^{2}(\Omega) .
\end{aligned}
$$

Therefore, for all $u \in C_{0}^{\infty}(\Omega)$ we have

$$
\|T u\|_{L^{2}(\Omega)} \leq \frac{1}{n}\left\|\Delta_{H} u\right\|_{L^{2}(\Omega)} .
$$


In the following we will use the fact that the formal adjoint of $X_{k}$ is $-X_{k}$. Let $u \in C_{0}^{\infty}(\Omega)$. For $k \in\{1, \ldots, n\}$ and $j \neq k+n, X_{k}$ and $X_{j}$ commute; therefore,

$$
\int_{\Omega}\left(X_{k} X_{j} u(x)\right)^{2} d x=\int_{\Omega} X_{k} X_{k} u(x) \cdot X_{j} X_{j} u(x) d x .
$$

For $j=k+n$ we have

$$
\begin{aligned}
& \int_{\Omega}\left(X_{k} X_{j} u(x)\right)^{2} d x=\int_{\Omega} X_{k} X_{j} u(x) \cdot\left(X_{j} X_{k} u(x)+T u(x)\right) d x \\
= & \int_{\Omega} X_{k} X_{j} u(x) \cdot X_{j} X_{k} u(x) d x+\int_{\Omega} X_{k} X_{j} u(x) \cdot T u(x) d x \\
= & -\int_{\Omega} X_{j} u(x) \cdot X_{k} X_{j} X_{k} u(x) d x+\int_{\Omega} X_{k} X_{j} u(x) \cdot T u(x) d x \\
= & -\int_{\Omega} X_{j} u(x) \cdot\left(X_{j} X_{k}+T\right) X_{k} u(x) d x+\int_{\Omega} X_{k} X_{j} u(x) \cdot T u(x) d x \\
= & -\int_{\Omega} X_{j} u(x) \cdot X_{j} X_{k} X_{k} u(x) d x+2 \int_{\Omega} X_{k} X_{j} u(x) \cdot T u(x) d x \\
= & \int_{\Omega} X_{k} X_{k} u(x) \cdot X_{j} X_{j} u(x) d x+2 \int_{\Omega} X_{k} X_{j} u(x) \cdot T u(x) d x .
\end{aligned}
$$

Similarly,

$$
\begin{aligned}
& \int_{\Omega}\left(X_{j} X_{k} u(x)\right)^{2} d x \\
= & \int_{\Omega} X_{k} X_{k} u(x) \cdot X_{j} X_{j} u(x) d x-2 \int_{\Omega} X_{j} X_{k} u(x) \cdot T u(x) d x .
\end{aligned}
$$

Therefore,

$$
\begin{aligned}
& \left\|X^{2} u\right\|_{L^{2}(\Omega)}^{2}=\sum_{k, j=1}^{2 n}\left\|X_{k} X_{j} u\right\|_{L^{2}(\Omega)}^{2} \\
= & \sum_{k, j=1}^{2 n} \int_{\Omega} X_{k} X_{k} u(x) \cdot X_{j} X_{j} u(x) d x+2 \sum_{k=1}^{n} \int_{\Omega}\left[X_{k}, X_{k+n}\right] u(x) \cdot T u(x) d x \\
= & \int_{\Omega}\left(\sum_{k=1}^{2 n} X_{k} X_{k} u(x)\right)^{2} d x+2 n \int_{\Omega}(T u(x))^{2} d x \\
\leq & \left(1+2 n \frac{1}{n^{2}}\right)\left\|\Delta_{H} u\right\|_{L^{2}(\Omega)}^{2}=\left(1+\frac{2}{n}\right)\left\|\Delta_{H} u\right\|_{L^{2}(\Omega)}^{2} .
\end{aligned}
$$

The constant $\sqrt{1+\frac{2}{n}}$ is sharp when $\Omega=\mathbb{H}^{n}$, because for $v=\Phi_{\lambda, 0,1}$ we have $T v=\frac{i}{n} \Delta_{H} v$.

\section{Cordes CONDitions FOR SECOND-ORder SUbelliptic PDE OPERATORS} IN NON-DIVERGENCE FORMS WITH MEASURABLE COEFFICIENTS

Let us consider now

$$
\mathcal{A} u=\sum_{i, j=1}^{2 n} a_{i j}(x) X_{i} X_{j} u
$$


where the functions $a_{i j} \in L^{\infty}(\Omega)$. Let us denote by $A=\left(a_{i j}\right)$ the $2 n \times 2 n$ matrix of coefficients.

Definition 2.1 ([2, 11]). We say that $A$ satisfies the Cordes condition $K_{\varepsilon, \sigma}$ if there exist $\varepsilon \in(0,1]$ and $\sigma>0$ such that

$$
0<\frac{1}{\sigma} \leq \sum_{i, j=1}^{2 n} a_{i j}^{2}(x) \leq \frac{1}{2 n-1+\varepsilon}\left(\sum_{i=1}^{2 n} a_{i i}(x)\right)^{2} \text {, a.e. } x \in \Omega .
$$

Theorem 2.1. Let $0<\varepsilon \leq 1, \sigma>0$ such that $\gamma=\sqrt{1-\varepsilon} c_{n}<1$ and A satisfies the Cordes condition $K_{\varepsilon, \sigma}$. Then for all $u \in H W_{0}^{2,2}(\Omega)$ we have

$$
\left\|X^{2} u\right\|_{L^{2}} \leq \sqrt{1+\frac{2}{n}} \frac{1}{1-\gamma}\|\alpha\|_{L^{\infty}}\|\mathcal{A} u\|_{L^{2}}
$$

where

$$
\alpha(x)=\frac{\langle A(x), I\rangle}{\|A(x)\|^{2}} .
$$

Proof. We denote by $I$ the identity $2 n \times 2 n$ matrix, by $\langle A, B\rangle=\sum_{i, j=1}^{2 n} a_{i j} b_{i j}$ the inner product and by $\|A\|=\sqrt{\sum_{i, j=1}^{2 n} a_{i j}^{2}}$ the Euclidean norm in $\mathbb{R}^{2 n \times 2 n}$ for matrices $A$ and $B$. The Cordes condition $K_{\varepsilon, \sigma}$ implies that

$$
\frac{\langle A(x), I\rangle^{2}}{\|A(x)\|^{2}} \geq 2 n-(1-\varepsilon)
$$

for all $x \in \Omega^{\prime} \subset \Omega$, where the Lebesgue measure of $\Omega \backslash \Omega^{\prime}$ is 0 .

Now let $x \in \Omega^{\prime}$ be arbitrary, but fixed. Consider the quadratic polynomial

$$
P(\alpha)=\|A(x)\|^{2} \alpha^{2}-2\langle A(x), I\rangle \alpha+2 n-(1-\varepsilon) .
$$

Inequality (2.3) shows that

$$
\min _{\alpha \in \mathbb{R}} P(\alpha)=P\left(\frac{\langle A(x), I\rangle}{\|A(x)\|^{2}}\right) \leq 0 .
$$

Therefore there exists

$$
\alpha(x)=\frac{\langle A(x), I\rangle}{\|A(x)\|^{2}}
$$

such that $P(\alpha(x)) \leq 0$. Observing that

$$
\|I-\alpha(x) A(x)\|^{2}=\|A(x)\|^{2} \alpha^{2}(x)-2\langle A(x), I\rangle \alpha(x)+2 n
$$

we get that (2.4) implies that

$$
\|I-\alpha(x) A(x)\|^{2} \leq 1-\varepsilon,
$$

which is equivalent to

$$
|\langle I-\alpha(x) A(x), M\rangle| \leq \sqrt{1-\varepsilon}\|M\| \text {, for all } M \in \mathcal{M}_{2 n}(\mathbb{R}) .
$$

Condition (2.6) can also be written as

$$
\left|\sum_{i=1}^{n} m_{i i}-\alpha(x) \sum_{i, j=1}^{n} a_{i j}(x) m_{i j}\right| \leq \sqrt{1-\varepsilon}\left(\sum_{i, j=1}^{n} m_{i j}^{2}\right)^{1 / 2}
$$

for all $M \in \mathcal{M}_{2 n}(\mathbb{R})$. 
Formula (2.7) and Lemma 1.1 imply that for all $u \in H W_{0}^{2,2}(\Omega)$ we have

$$
\begin{gathered}
\int_{\Omega}\left|\Delta_{H} u(x)-\alpha(x) \mathcal{A} u(x)\right|^{2} d x \leq(1-\varepsilon) \int_{\Omega} \sum_{i, j=1}^{2 n}\left(X_{i} X_{j} u(x)\right)^{2} d x \\
\leq(1-\varepsilon) c_{n}^{2} \int_{\Omega}\left|\Delta_{H} u(x)\right|^{2} d x .
\end{gathered}
$$

Therefore, for $\gamma=\sqrt{1-\varepsilon} c_{n}<1$ we get

$$
\left\|\Delta_{H} u-\alpha \mathcal{A} u\right\|_{L^{2}(\Omega)} \leq \gamma\left\|\Delta_{H} u\right\|_{L^{2}(\Omega)},
$$

which shows that

$$
\begin{gathered}
\left\|X^{2} u\right\|_{L^{2}(\Omega)} \leq c_{n}\left\|\Delta_{H} u\right\|_{L^{2}(\Omega)} \\
\leq \frac{c_{n}}{1-\gamma}\|\alpha \mathcal{A} u\|_{L^{2}(\Omega)} \leq \frac{c_{n}}{1-\gamma}\|\alpha\|_{L^{\infty}(\Omega)}\|\mathcal{A} u\|_{L^{2}(\Omega)} .
\end{gathered}
$$

3. $H W^{2,2}$-INTERIOR REGULARITY FOR P-HARMONIC FUNCTIONS IN $\mathbb{H}^{n}$

Let $\Omega \in \mathbb{H}^{n}$ be a domain, $h \in H W^{1, p}(\Omega)$ and $p>1$. Consider the problem of minimizing the functional

$$
\Phi(u)=\int_{\Omega}|X u(x)|^{p} d x
$$

over all $u \in H W^{1, p}(\Omega)$ such that $u-h \in H W_{0}^{1, p}(\Omega)$. The Euler equation for this problem is the p-Laplace equation

$$
\sum_{i=1}^{2 n} X_{i}\left(|X u|^{p-2} X_{i} u\right)=0, \text { in } \Omega
$$

A function $u \in H W^{1, p}(\Omega)$ is called a weak solution for (3.1) if

$$
\sum_{i=1}^{2 n} \int_{\Omega}|X u(x)|^{p-2} X_{i} u(x) \cdot X_{i} \varphi(x) d x=0, \forall \varphi \in H W_{0}^{1, p}(\Omega) .
$$

$\Phi$ is a convex functional on $H W^{1, p}$; therefore weak solutions are minimizers for $\Phi$ and vice-versa.

For $m \in \mathbb{N}$ let us now define the approximating problems of minimizing the functionals

$$
\Phi_{m}(u)=\int_{\Omega}\left(\frac{1}{m}+|X u(x)|^{2}\right)^{\frac{p}{2}}
$$

and the corresponding Euler equations

$$
\sum_{i=1}^{2 n} X_{i}\left(\left(\frac{1}{m}+|X u|^{2}\right)^{\frac{p-2}{2}} X_{i} u\right)=0, \text { in } \Omega .
$$

The weak form of this equation is

$$
\sum_{i=1}^{2 n} \int_{\Omega}\left(\frac{1}{m}+|X u(x)|^{2}\right)^{\frac{p-2}{2}} X_{i} u(x) \cdot X_{i} \varphi(x) d x=0, \text { for all } \varphi \in H W_{0}^{1, p}(\Omega) .
$$


The differentiated version of equation (3.3) has the form

$$
\sum_{i, j=1}^{2 n} a_{i j}^{m} X_{i} X_{j} u=0, \text { in } \Omega
$$

where

$$
a_{i j}^{m}(x)=\delta_{i j}+(p-2) \frac{X_{i} u(x) X_{j} u(x)}{\frac{1}{m}+|X u(x)|^{2}} .
$$

Let us consider a weak solution $u_{m} \in H W^{1, p}(\Omega)$ of equation (3.3). Then $a_{i j}^{m} \in$ $L^{\infty}(\Omega)$. Define the mapping $L_{m}: W_{0}^{2,2}(\Omega) \rightarrow L^{2}(\Omega)$ by

$$
L_{m}(v)(x)=\sum_{i, j=1}^{2 n} a_{i j}^{m}(x) X_{i} X_{j} v(x) .
$$

We will check the validity of Theorem 2.1 for $L_{m}$. We have

$$
\sum_{i=1}^{2 n} a_{i i}^{m}(x)=2 n+(p-2) \frac{\left|X u_{m}\right|^{2}}{\frac{1}{m}+\left|X u_{m}\right|^{2}}
$$

and

$$
\sum_{i, j=1}^{2 n}\left(a_{i j}^{m}(x)\right)^{2}=2 n+2(p-2) \frac{\left|X u_{m}\right|^{2}}{\frac{1}{m}+\left|X u_{m}\right|^{2}}+(p-2)^{2} \frac{\left|X u_{m}\right|^{4}}{\left(\frac{1}{m}+\left|X u_{m}\right|^{2}\right)^{2}} .
$$

Denote

$$
(p-2) \frac{\left|X u_{m}\right|^{2}}{\frac{1}{m}+\left|X u_{m}\right|^{2}}=\Lambda .
$$

Therefore, for an $\varepsilon \in\left(1-\frac{1}{c_{n}^{2}}, 1\right)$ we need

$$
2 n+2 \Lambda+\Lambda^{2} \leq \frac{1}{2 n-1+\varepsilon}(2 n+\Lambda)^{2} .
$$

This leads to

$$
\begin{gathered}
(2 n-1) \Lambda^{2} \leq(1-\varepsilon)\left(2 n+2 \Lambda+\Lambda^{2}\right) \\
<\frac{1}{c_{n}^{2}}\left(2 n+2 \Lambda+\Lambda^{2}\right)
\end{gathered}
$$

Hence,

$$
\left((2 n-1) c_{n}^{2}-1\right) \Lambda^{2}-2 \Lambda-2 n<0
$$

Solving this inequality we get

$$
\Lambda \in\left(\frac{1-\sqrt{2 n\left((2 n-1) c_{n}^{2}-1\right)+1}}{(2 n-1) c_{n}^{2}-1}, \frac{1+\sqrt{2 n\left((2 n-1) c_{n}^{2}-1\right)+1}}{(2 n-1) c_{n}^{2}-1}\right) .
$$

Using $c_{n}^{2}=\frac{n+2}{n}$ and the fact that $\frac{\left|X u_{m}\right|^{2}}{\frac{1}{m}+\left|X u_{m}\right|^{2}}<1$ for all $m \in \mathbb{N}$ we have that

$$
p-2 \in\left(\frac{n-n \sqrt{4 n^{2}+4 n-3}}{2 n^{2}+2 n-2}, \frac{n+n \sqrt{4 n^{2}+4 n-3}}{2 n^{2}+2 n-2}\right)
$$

and that the operators $L_{m}$ satisfy the assumptions of Theorem 2.1 uniformly in $m$.

Let us remark that in the case $n=1$ we have

$$
p-2 \in\left(\frac{1-\sqrt{5}}{2}, \frac{1+\sqrt{5}}{2}\right) \text {. }
$$


Theorem 3.1. Let

$$
2 \leq p<2+\frac{n+n \sqrt{4 n^{2}+4 n-3}}{2 n^{2}+2 n-2} .
$$

If $u \in H W^{1, p}(\Omega)$ is a minimizer for the functional $\Phi$, then $u \in H W_{\mathrm{loc}}^{2,2}(\Omega)$.

Proof. The case $p=2$ is well known, so let us suppose $p \neq 2$. Let $u \in H W^{1, p}(\Omega)$ be a minimizer for $\Phi$. Consider $x_{0} \in \Omega$ and $r>0$ such that $B_{4 r}=B\left(x_{0}, 4 r\right) \subset \subset \Omega$. We need a cut-off function $\eta \in C_{0}^{\infty}\left(B_{2 r}\right)$ such that $\eta=1$ on $B_{r}$. Also consider minimizers $u_{m}$ for $\Phi_{m}$ on $H W^{1, p}\left(B_{2 r}\right)$ subject to $u_{m}-u \in H W_{0}^{1, p}\left(B_{2 r}\right)$. Then $u_{m} \rightarrow u$ in $H W^{1, p}\left(B_{2 r}\right)$ as $m \rightarrow \infty$.

By [3, 7] we get that for $2 \leq p<4$ we have $u_{m} \in H W_{\text {loc }}^{2,2}(\Omega)$, but with bounds depending on $m$, and also that $u_{m}$ satisfies the equation $L_{m}\left(u_{m}\right)=0$ a.e. in $B_{2 r}$. So, in $B_{2 r}$ we have a.e.

$$
X_{i} X_{j}\left(\eta^{2} u_{m}\right)=X_{i} X_{j}\left(\eta^{2}\right) u_{m}+X_{j}\left(\eta^{2}\right) X_{i} u_{m}+X_{i}\left(\eta^{2}\right) X_{j} u_{m}+\eta^{2} X_{i} X_{j} u_{m}
$$

and hence

$$
L_{m}\left(\eta^{2} u_{m}\right)=u_{m} L_{m, u_{m}}\left(\eta^{2}\right)+\sum_{i, j=1}^{2 n} a_{i j}^{m}(x)\left(X_{j}\left(\eta^{2}\right) X_{i} u_{m}+X_{i}\left(\eta^{2}\right) X_{j} u_{m}\right) .
$$

By Theorem 2.1 it follows that

$$
\begin{aligned}
\left\|X^{2} u_{m}\right\|_{L^{2}\left(B_{r}\right)} & \leq\left\|X^{2}\left(\eta^{2} u_{m}\right)\right\|_{L^{2}\left(B_{2 r}\right)} \leq c\left\|L_{m}\left(\eta^{2} u_{m}\right)\right\|_{L^{2}\left(B_{2 r}\right)} \\
& \leq c\left\|u_{m}\right\|_{H W^{1, p}\left(B_{2 r}\right)} \leq c\|u\|_{H W^{1, p}\left(B_{2 r}\right)}
\end{aligned}
$$

where $c$ is independent of $m$. Therefore, $u \in H W^{2,2}\left(B_{r}\right)$.

Remark 3.1. Observe that the range for $p$ given by Theorem 3.1 is shrinking from $\left[2, \frac{5+\sqrt{5}}{2}\right)$ to $[2,3]$ as $n$ increases from 1 to $\infty$.

For the case $p<2$ we need the following lemmas. The first lemma is an interpolation result and its proof is based on integration by parts.

Lemma 3.1. For all $u \in C_{0}^{\infty}(\Omega)$ and for all $\delta>0$ there exists $c(\delta)>0$ such that

$$
\|X u\|_{L^{2}(\Omega)}^{2} \leq \delta\left\|X^{2} u\right\|_{L^{2}(\Omega)}^{2}+c(\delta)\|u\|_{L^{2}(\Omega)}^{2} .
$$

Proof.

$$
\begin{aligned}
\|X u\|_{L^{2}(\Omega)}^{2} & =\sum_{i=1}^{2 n} \int_{\Omega} X_{i} u(x) X_{i} u(x) d x=-\sum_{i=1}^{2 n} \int_{\Omega} u(x) X_{i} X_{i} u(x) d x \\
& =-\int_{\Omega} u(x) \Delta_{H} u(x) d x \leq \frac{\delta}{2 n} \int_{\Omega}\left|\Delta_{H} u(x)\right|^{2} d x+c(\delta) \int_{\Omega} u^{2}(x) d x \\
& \leq \delta \int_{\Omega}\left|X^{2} u(x)\right|^{2} d x+c(\delta) \int_{\Omega} u^{2}(x) d x .
\end{aligned}
$$

From Lemma 3.1 and the higher-order extension results available for the Sobolev spaces on the Heisenberg group [6, 9] we get the following result.

Lemma 3.2. For all $u \in H W^{2,2}\left(B_{r}\right)$ and all $\delta>0$ there exists $c(\delta)>0$ such that

$$
\|X u\|_{L^{2}\left(B_{r}\right)}^{2} \leq \delta\left\|X^{2} u\right\|_{L^{2}\left(B_{r}\right)}^{2}+c(\delta)\|u\|_{L^{2}\left(B_{r}\right)}^{2} .
$$


By Lemmas 3.1 and 3.2 we can use a method similar to the proof of Theorem 9.11 [5] to get the following result.

Lemma 3.3. Let us suppose that the operator $\mathcal{A}$ satisfies the assumptions of Theorem 4.1 and that $B_{3 r} \subset \Omega$. Then

$$
\left\|X^{2} u\right\|_{L^{2}\left(B_{r}\right)} \leq c\left(\|\mathcal{A} u\|_{L^{2}\left(B_{2 r}\right)}+\|u\|_{L^{2}\left(B_{2 r}\right)}\right)
$$

for all $u \in H W_{\mathrm{loc}}^{2,2}\left(B_{3 r}\right)$.

Proof. Let $\eta \in C_{0}^{\infty}\left(B_{2 r}\right), 0<\sigma<1$ and $\sigma^{\prime}=\frac{1+\sigma}{2}$ such that $\eta$ is a cut-off function between $B_{\sigma 2 r}$ and $B_{\sigma^{\prime} 2 r}$ satisfying

$$
|X \eta| \leq \frac{4}{(1-\sigma) r} \text { and }\left|X^{2} \eta\right| \leq \frac{16}{(1-\sigma)^{2} r^{2}} .
$$

Then we can use Theorem 2.1 for $\eta u$ to get

$$
\begin{aligned}
& \left\|X^{2} u\right\|_{L^{2}\left(B_{\sigma 2 r}\right)} \leq\left\|X^{2}(\eta u)\right\|_{L^{2}\left(B_{2 r}\right)} \leq c\|\mathcal{A}(\eta u)\|_{L^{2}\left(B_{2 r}\right)} \\
& \leq c\left\|\eta \mathcal{A} u+u \mathcal{A}(\eta)+\sum_{i, j=1}^{2 n} a_{i j}\left(X_{j}(\eta) X_{i} u+X_{i}(\eta) X_{j} u\right)\right\|_{L^{2}\left(B_{2 r}\right)} \\
& \leq c\left(\|\mathcal{A} u\|_{L^{2}\left(B_{2 r}\right)}+\frac{1}{(1-\sigma) r}\|X u\|_{L^{2}\left(B_{\sigma^{\prime} 2 r}\right)}+\frac{1}{(1-\sigma)^{2} r^{2}}\|u\|_{L^{2}\left(B_{\sigma^{\prime} 2 r}\right)}\right) .
\end{aligned}
$$

For $k \in\{0,1,2\}$ let us use the seminorms

$$
\|\| u\|\|_{k}=\sup _{0<\sigma<1}(1-\sigma)^{k} r^{k}\left\|X^{k} u\right\|_{L^{2}\left(B_{\sigma 2 r}\right)} .
$$

Then

$$
\|\| u \|_{2} \leq c\left(r^{2}\|\mathcal{A} u\|_{L^{2}\left(B_{2 r}\right)}+\|\| u\|\|_{1}+\|\| u\|\|_{0}\right) .
$$

Lemma 3.2 implies that for $\delta>0$ small we have

$$
\left\|\left|u \left\|_{1} \leq \delta\left|\left\|u \left|\left\|_{2}+c(\delta)\left|\|u \mid\|_{0}\right.\right.\right.\right.\right.\right.\right.\right.
$$

Therefore,

$$
\|\mid u\|_{2} \leq c\left(r^{2}\|\mathcal{A} u\|_{L^{2}\left(B_{2 r}\right)}+\|\| u \|_{0}\right)
$$

and hence

$$
\left\|X^{2} u\right\|_{L^{2}\left(B_{\sigma 2 r}\right)} \leq \frac{c}{(1-\sigma)^{2} r^{2}}\left(r^{2}\|\mathcal{A} u\|_{L^{2}\left(B_{2 r}\right)}+\|u\|_{L^{2}\left(B_{2 r}\right)}\right) .
$$

For $\sigma=\frac{1}{2}$ we get the desired inequality.

Theorem 3.2. Let us consider the Heisenberg group $\mathbb{H}^{1}$ and

$$
\frac{\sqrt{17}-1}{2} \leq p \leq 2 \text {. }
$$

If $u \in H W^{1, p}(\Omega)$ is a minimizer for the functional $\Phi$, then $u \in H W_{\mathrm{loc}}^{2,2}(\Omega)$. 
Proof. We start the proof in the same way as we did in the proof of Theorem 3.1. Let $u \in H W^{1, p}(\Omega)$ be a minimizer for $\Phi$. Consider $x_{0} \in \Omega$ and $r>0$ such that $B_{4 r}=B\left(x_{0}, 4 r\right) \subset \subset \Omega$. We need a test function $\eta \in C_{0}^{\infty}\left(B_{3 r}\right)$. Also consider minimizers $u_{m}$ for $\Phi_{m}$ on $H W^{1, p}\left(B_{3 r}\right)$ subject to $u_{m}-u \in H W_{0}^{1, p}\left(B_{3 r}\right)$. Then $u_{m} \rightarrow u$ in $H W^{1, p}\left(B_{3 r}\right)$ as $m \rightarrow \infty$. We use the facts that

$$
\frac{4}{3}<\frac{5-\sqrt{5}}{2}<\frac{\sqrt{17}-1}{2}<2,
$$

the homogeneous dimension of $\mathbb{H}^{1}$ is $Q=4$, and

$$
2 \leq \frac{4 p}{4-p} \text { for all } \frac{4}{3} \leq p<2 .
$$

The Sobolev embeddings result in the subelliptic setting [1] says that

$$
H W_{0}^{1, p}\left(B_{3 r}\right) \hookrightarrow L^{q}\left(B_{3 r}\right), \text { for } 1 \leq q \leq \frac{4 p}{4-p} .
$$

Therefore, $u_{m} \rightarrow u$ in $L^{2}\left(B_{3 r}\right)$. Also, using that (see [3]) for $\frac{\sqrt{17}-1}{2} \leq p \leq 2$ we have $u_{m} \in H W_{\text {loc }}^{2, p}\left(B_{3 r}\right)$ we get that $X u_{m} \in L_{\text {loc }}^{2}\left(B_{3 r}\right)$. Let us remark that these bounds of $X^{2} u_{m}$ in $L^{p}$ may depend on $m$ and that $L_{m}\left(u_{m}\right)=0$ a.e. in $B_{3 r}$. Moreover,

$$
\begin{gathered}
\left\|L_{m}\left(\eta^{2} u_{m}\right)\right\|_{L^{2}\left(B_{3 r}\right)} \\
\leq c\left(\left\|u_{m}\right\|_{L^{2}(\operatorname{Supp} \eta)}+\left\|X u_{m}\right\|_{L^{2}(\operatorname{Supp} \eta)}\right)<+\infty,
\end{gathered}
$$

and hence $u_{m} \in H W_{\text {loc }}^{2,2}\left(B_{3 r}\right)$. By Lemma 3.3 for all $m$ sufficiently large we have

$$
\left\|X^{2}\left(u_{m}\right)\right\|_{L^{2}\left(B_{r}\right)} \leq c\left\|u_{m}\right\|_{L^{2}\left(B_{2 r}\right)} \leq 2 c\|u\|_{L^{2}\left(B_{2 r}\right)},
$$

which shows that $X^{2} u_{m}$ is uniformly bounded in $H W^{2,2}\left(B_{r}\right)$; hence $u \in$ $H W^{2,2}\left(B_{r}\right)$.

In the forthcoming article [4] we establish the $C^{1, \alpha}$ regularity for p-harmonic functions in $\mathbb{H}^{n}$ when $p$ is in a neighborhood of 2 .

\section{ACKNOWLEDGEMENT}

The authors would like to thank Robert S. Strichartz for his valuable hint regarding the spectral analysis of the sublaplacian and estimate (1.3), and Silvana Marchi for making available her papers and for her conversations with the second author.

\section{REFERENCES}

[1] L. Capogna, D. Danielli and N. Garofalo, An embedding theorem and the Harnack inequality for nonlinear subelliptic equations, Comm. Partial Differential Equations, 18(1993), 17651794. MR1239930 (94j:35038)

[2] H. O. Cordes, Zero order a priori estimates for solutions of elliptic differential equations, Proceedings of Symposia in Pure Mathematics IV (1961). MR0146511 (26:4033)

[3] A. Domokos, Differentiability of solutions for the non-degenerate p-Laplacian in the Heisenberg group, J. Differential Equations 204(2004), 439-470. 
[4] A. Domokos and Juan J. Manfredi, $C^{1, \alpha}$-regularity for p-harmonic functions in the Heisenberg group for $p$ near 2, to appear in The p-harmonic equation and recent advances in analysis, Contemporary Mathematics, editor Pietro Poggi-Corradini.

[5] D. Gilbarg and N.S. Trudinger, Elliptic Partial Differential Equations of Second Order, Springer-Verlag, 1983. MR0737190 (86c:35035)

[6] G. Lu, Polynomials, higher order Sobolev extension theorems and interpolation inequalities on weighted Folland-Stein spaces on stratified groups, Acta Math. Sinica, English Series, 16(2000), 405-444. MR1787096 (2001k:46054)

[7] S. Marchi, $C^{1, \alpha}$ local regularity for the solutions of the $p$-Laplacian on the Heisenberg group for $2 \leq p \leq 1+\sqrt{5}$, Z. Anal. Anwendungen 20(2001), 617-636. See Erratum, Z. Anal. Anwendungen 22 (2003), 471-472. MR1863937 (2002i:35037); MR2000279 (2004g:35034)

[8] S. Marchi, $C^{1, \alpha}$ local regularity for the solutions of the p-Laplacian on the Heisenberg group. The case $1+\frac{1}{\sqrt{5}}<p \leq 2$, Comment. Math. Univ. Carolinae 44(2003), 33-56. See Erratum, to appear. MR2045844

[9] D. Nhieu, Extension of Sobolev spaces on the Heisenberg group, C. R. Acad. Sci. Ser. I Math., 321(1995), 1559-1564. MR 1367807 (98c:22011)

[10] R. S. Strichartz, $L^{p}$ Harmonic analysis and Radon transforms on the Heisenberg group, J. Funct. Analysis, 96(1991), 350-406. MR.1101262(92d:22015)

[11] G. Talenti, Sopra una classe di equazioni ellitiche a coeficienti misurabili, Ann. Mat. Pura. Appl. 69(1965), 285 - 304. MR0201816 (34:1698)

Department of Mathematics, University of Pittsburgh, 301 Thackeray Hall, PittsBurgh, Pennsylvania 15260

Current address: Department of Mathematics and Statistics, California State University Sacramento, 6000 J Street, Sacramento, California 95819

E-mail address: domokos@csus.edu

Department of Mathematics, University of Pittsburgh, 301 Thackeray Hall, PittsBurgh, Pennsylvania 15260

E-mail address: manfredi@pitt.edu 